\title{
A Path Planning Method for Substation Laser Inspection Robot Based on Improved Ant Colony Algorithm
}

\author{
Yan Deng ${ }^{1, a,{ }^{*}}$, Hongyu Hu ${ }^{2, b}$, Xiaolu Tian ${ }^{1, c}$, Jiake Liü ${ }^{1, d}$, Mingyang Liu ${ }^{3, \text { e }}$ and \\ Yafei Wang ${ }^{1, \mathrm{f}}$ \\ ${ }^{1}$ Shandong Luneng Intelligence Technology co., LTD, No.2008, Xinluo Street, Jinan City, Shandong \\ Province, China \\ 2Jiangxi Electric Power Company, No.666, Hubin East Road, Nanchang City, Jiangxi Province, \\ China \\ ${ }^{3}$ Lanzhou University, No.222, Tianshui South Road, Lanzhou City, Gansu Province, China \\ a sunnygirlD_Y@163.com, b hongyuhunc@163.com, ctxl_01@126.com, d liujiake9768@163.com, \\ eliu_sharon@icloud.com, ${ }^{\dagger}$ wangyafei@163.com
}

Keywords: Laser Inspection Robot, Path plan, Topology, Undirected Graph, Ant Colony Algorithm, Dijkstra Algorithm.

\begin{abstract}
In order to shorten the inspection path and time-consuming, improve the intelligence degree of the laser inspection robot and apply to the large-scale TSP in the substation, the paper proposes a path planning method based on the improved ant colony algorithm, the navigation method, the walking characteristics, the inspection task and the road environment. This method uses the topological method to model the road environment. The Dijkstra algorithm and the improved ant colony algorithm are used to plan the inspection path of the laser inspection robot. In this way, the laser inspection robot can re-plan the new inspection path for the remaining undetected task points after encountering an obstacle, and finally return to the starting point. In this paper, Visual Studio $\mathrm{C}++$ is used to test the improved ant colony algorithm and Greedy algorithm. The results show that the improved ant colony algorithm has obvious advantages in path length and the number of passing points, which can realize that the inspection route of the laser inspection robot is optimal or sub-optimal. At the same time, the dynamic simulation of the method is carried out based on MATLAB. The simulation results show that the method can realize self-real-timely avoid obstacle and re-plan the new inspection route after encountering an obstacle. It can ensure the completion of the inspection task. And the method has good applicability, robustness and high efficiency.
\end{abstract}

\section{Introduction}

Based on the intelligent robot technology, the substation inspection robot system use the intelligent robot body to autonomously move, dock according to the inspection task in a specific location, and detect the substation instrumentation equipment by the carrying infrared camera, visible CCD, and so on. In this way, it can effectively detect the internal thermal defects, external machinery and electrical and other issues for electrical equipment. So as to it can improve the efficiency and quality of work, and truly play a role in the reduction of efficiency, and ultimately promote the substation unattended development ${ }^{[1-2]}$. The path planning is the basis of intelligent robots that it can efficiently carry out inspection tasks. The pros and cons of path planning affect the intelligence and performance of inspection robots. Therefore, it is necessary to study the path planning of intelligent inspection robots in substations.

The path planning of substation inspection robot is a non-complete movement planning. It needs to consider the limited DOF of the robot system, provides the current point and the stop point that the robot must go through for input interface, makes sure each task point will arrive and successfully completes the inspection task ${ }^{[1]}$. The purpose of the path planning is to find an optimal or sub-optimal route for the intelligent inspection robot. The route must start from the current location of the robot, and go through all the selected points that need to perform detection tasks. Meantime it makes the 
robot could able to autonomously avoid obstacle in real time and re-plan the inspection path after encountering obstacles. And the point to be detected is the stop point where the robot in the substation needs to identify the substation equipment on the inspection route.

At present, the research on the path planning of the inspection robot in the substation is mainly attach to the magnetic track, laser, GPS, visual navigation and so on. In the substation environment, GPS positioning is susceptible to the environment, and the accuracy can only reach meters ${ }^{[3]}$. The running direction of the magnetic track navigation is not flexible and too stable. Visual navigation is susceptible to light and not suitable for robots in the outdoor environment.

At present, there are two shortcomings of the path planning method of the laser inspection robot in the substation. The first, the path programmed by the global path planning algorithm is not optimal or sub-optimal, and it is time consuming to plan path for the large number of task points. The second, the current path planning algorithms do not take into account the temporary obstacles that encounter during the inspection. When the laser inspection robot encounter an obstacle, it can't independently re-plan the inspection path, but stop before the obstacle and wait for the staff to handle. In literature [1], the improved Dijkstra algorithm and the simulated annealing algorithm are used to plan the inspection path. The method is implemented in a barrier-free environment. And when the inspection robot encounters an obstacle, it will stop and wait for the staff to deal with. At the same time, the test of the algorithm is based on a small number of points, it is suitable for small-scale TSP and not suitable for large-scale TSP. Based on the actual application environment, navigation technology and inspection task of the laser inspection robot, this paper proposed a combined method of Dijkstra algorithm and improved ant colony algorithm to plan the inspection path for the laser inspection robot.

\section{Environmental Modeling}

Map modeling based on real environment is critical for the path planning of mobile robots. The map modeling is abstract the physical environment of the actual work into a mathematical model, and provide environmental information for the path planning algorithm of the laser inspection robot. According to the working environment and working way of the substation laser inspection robot, the paper choose the topology method to model the relationship between the turning point and the detection point in the map environment, and obtain an undirected topological map.

Topology method ${ }^{[4-5]}$ abstract the map environment as a structure diagram with topological meaning. In the topology, each node represents a subspace in which the characteristics are consistent in the environment map. As shown in Figure 1, the topological relation between the feature nodes is equivalent to the pass-through path of the map subspace. In Figure 1, the node is represented by a white circle, the number in the circle is the number of the node, and the number on the line between adjacent nodes is the actual distance.

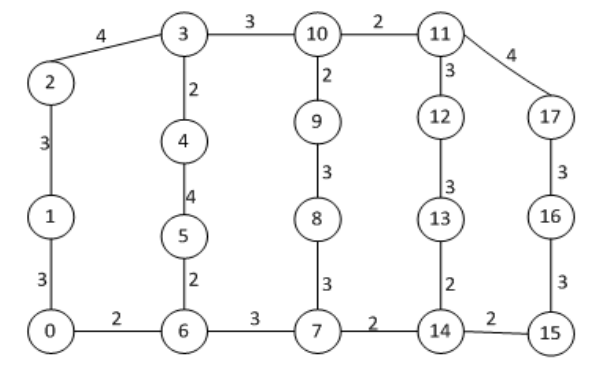

Figure 1. The Diagram of Topology

Based on the actual road environment and laser navigation mode of the laser inspection robot in the substation, the labeling of nodes in this paper is divided into two types. The first type is the real detection point that the laser inspection robot needs to dock and perform inspection tasks, such as instrumentation detection, sound detection and other tasks. The second type is the dummy test point, that is, the turning points. This type of node only need to dock and needn't to perform inspection tasks for the laser inspection robot. This type of node is mainly used to guide the laser inspection robot to 
change the direction of movement, such as straight, reverse, turn, U-turn, and so on. When encounter obstacles, it helps to update the topology in real time. This paper creates topology structure for all the detection points and the turning points in the map, and obtains the topology matrix. In the topology matrix, the distance between two directly adjacent points is the actual distance of the working road, and the distance between two points that not directly adjacent is infinity.

\section{Path Planning}

The path planning of substation laser inspection robot is to find an optimal or sub-optimal inspection path that start from the starting point, go through all the points that need to be tested, and finally return to the charging room. At the same time, when laser inspection robot encounter an obstacle, it can firstly update the topology matrix between the nodes according to the position of the obstacle, and then re-plan the new path for the remaining undetected points, and complete the full inspection task.

The path planning of the substation laser inspection robot is a nonholonomic global path planning. The path planning can be divided into two parts. The first part is the local path, that is, to obtain the shortest path between any two points. The second part is the global path, that is, to obtain the optimal or sub-optimal combination of all the test points that to be tested.

\subsection{Local Path Planning}

The local path planning is to find a collision-free shortest path from the starting point to the target point and successfully avoid the obstacle. At present, many scholars have done a lot of research on the optimal path between two points. In literature [6], the author proposed a grid method to model the environment, and used the cellular neural network to plan an optimal path without collision in the static environment. In literature [7], the ant colony algorithm, Dijkstra algorithm and heuristic algorithm were used to plan the optimal path between two points. In literature [8], an improved artificial potential method was used to plan the search path of mobile robots. Most of the algorithms mentioned in these references only validated the effectiveness of the algorithm and did not compare with other algorithms.

According to the practical application of the laser inspection robot in the substation and the characteristics of environmental modeling, this paper adopts the Dijkstra algorithm to plan the local path. The main feature of the Dijkstra algorithm ${ }^{[9]}$ is that it extends from the starting point to the outer layer and until it extends to the target point. Its basic idea is to set the vertex set $\mathrm{S}$ and continue to do greedy selection to expand $\mathrm{S}$.

In this paper, on the basis of the undirected topological map that have constructed, the Dijkstra algorithm is used to obtain the local optimal or sub-optimal paths between any two nodes. In the meantime, the path length of any two points is stored in the distance matrix, each row and column of the matrix corresponds to the number of the detection point. The matrix will be used in the global path planning. And the optimal path between any two nodes can be used to refine the specific path. The reason of refine the specific path is that the nodes in the optimal path between the detection points are not necessarily directly adjacent to each other, that is, there may be turning points or other detection points between two adjacent detection points in the final planned sequence.

\subsection{Global Path Planning}

Similar to the TSP (Travelling Salesman Problem), the substation laser inspection robot needs to detect all the task points in an optimal and non-repeating path, and complete the task of the inspection. Unlike the TSP, the path planning algorithm needs to make the laser inspection robot self-obstruction when encounter obstacles and autonomously re-plan the optimal path for the unselected points after avoiding the obstacle. In fact, before the robot encounter an obstacle, the optimal ranking of the task points is a combinatorial optimization problem. When the obstacle is encountered, the laser inspection robot will avoid obstruction by control information, update the topology between the nodes, and then re-plan the new path for the remaining task points, and finally return to the charging room. 
At present, there are many scholars have studied the global path planning method for the similar problems like TSP. In literature [1], an improved Dijkstra algorithm and simulated annealing algorithm were combined to plan path for robots. The algorithm could realize the basic path planning. But it didn't realize the real-time update of the path when encountered obstacles, and it was only applicable to magnetic navigation laser inspection robots. In order to overcome the shortcoming that easily fall into the local optimum in the large-scale TSP, the literature [10] proposed a group of intelligent hybrid algorithm which combined ant colony algorithm and particle swarm optimization algorithm. The algorithm had strong global optimization ability and good search convergence. But the simulation of the algorithm was based on the data that less than 200 cities. The simulation could not show that the algorithm had a better planning effect on the large-scale TSP. In literature [11], a hybrid algorithm was proposed for large-scale TSP. The algorithm improved the quality of the initial solution, reduced the computational complexity, and was suitable for the large-scale TSP. But the efficiency of solving TSP was limited. With the expansion of the scale, the algorithm required the longer time.

At present, there is no general method that meeting the various requirements for TSP. All the algorithms for TSP are based on the actual application requirements, and are put forward for solving the practical application problem. In the substation, the number of nodes is more, and the number of detection points in each inspection task ranges from tens to hundreds. In view of this practical application, this paper proposed a path planning method combining Dijkstra algorithm with improved ant colony algorithm. The improved ant colony algorithm in the global path planning was compared with the Greedy algorithm that applied in some current substations by testing and dynamic simulation. It was found that the improved ant colony algorithm had obvious advantages in path length and time efficiency when the number of the detection points were less than 400. Based on the comparison of them, the improved ant colony algorithm had obvious advantages. In order to meet the practical application scene, and take into account the time efficiency of the algorithm, this paper chooses the improved ant colony algorithm to solve the problem of the optimal combination in the global path planning.

\section{The Process of the Path Planning Algorithm}

According to the road environment and laser navigation mode of substation laser inspection robot, the road environment is modeled by topology method, and the path of the laser inspection robot is planned by local path planning and global path planning.

In order to easily update the topology between the detection points according to the position of the random obstacle, this paper chooses to set dock point at each intersection and distinguishes label with the detection point. At the same time, this paper adds the function of autonomy obstacle avoidance for the laser inspection robot. When the laser inspection robot encounters an obstacle, it can update the topology according to the positions of the robot and obstacle, then re-plan the check path for the remaining undetected points, and until back to the charge cottage. This algorithm improves the intelligence of the laser inspection robot.

Based on the Dijkstra algorithm and the improved ant colony algorithm, the path planning algorithm is divided into 13 steps.

Step 1: initialize the information that including the starting point, the ending point, the number of task points, the task point number, and read the shortest distance matrix and the nearest neighbor matrix of all points.

Step 2: obtain the corresponding shortest distance matrix according to the current task point number.

Step 3: use the improved ant colony algorithm that combined ant colony algorithm and neighborhood search algorithm to plan path, and obtain the better inspection path.

Step 4: use the nearest neighbor matrix to refine the task sequence, and ensure that the laser inspection robot can follow the path to correctly inspect and complete the inspection task. 
Step 5: in the process of patrolling according to the planned path sequence, the laser inspection robot uses the sensor carried by itself to detect the obstacle in real time. And if the obstacle is not encountered, it performs the step 12, otherwise performs the step 6.

Step 6: determine whether there are undetected task points. If there are some undetected task points, it performs the step 7, otherwise performs the step 13.

Step 7: obtain the current location of the laser inspection robot, update the topology between all points according to the location of the obstacle, obtain the nearest neighbor point of the laser inspection robot, take the point as the new starting point, and obtain the remaining undetected task points.

Step 8, determine whether there are isolated points. Namely, when the laser inspection robot encountered obstacles, it need to determine whether there are some unreachable task point. If there are some unreachable task point, it performs the step 9, otherwise performs the step 10.

Step 9, remove the isolated points, update the map information, get the new topological matrix that including all the remaining points, and then perform the step 11.

Step 10, update the map information, get the new topological matrix that including all points, and then perform the step 11 .

Step 11, use the Dijkstra algorithm to obtain the latest distance matrix and the nearest neighbor matrix, and then perform the step 2.

Step 12, determine whether the inspection is over. If the inspection is completed, the thirteenth step is executed, otherwise perform the step 5.

Step 13, the inspection is over and the robot waits for the next inspection task.

\section{Test Contrast and Simulation Results}

In the global path planning section, in order to compare the advantages and disadvantages of the improved ant colony algorithm and Greedy algorithm, this paper uses the Visual Studio C++ to simulate and test them. On the basis of the distance matrix and the nearest neighbor matrix that obtained by the Dijkstra algorithm under the barrierless environment, this paper compares them from the four dimensions of the path length, the number of passing points, the time consuming and whether the test results of 10 times are same. The simulation results are shown in Table 1. In Table 1, the G stands for the Greedy algorithm, and the ACO stands for the improved ant colony algorithm. 
Table 1. The Comparison Table of Greedy Algorithm and Improved Ant Colony Algorithm Global Path Planning Results

\begin{tabular}{|c|c|c|c|c|c|c|c|c|c|}
\hline $\begin{array}{c}\text { Task } \\
\text { number }\end{array}$ & $\begin{array}{l}\text { The } \\
\text { number } \\
\text { of task } \\
\text { points }\end{array}$ & $\begin{array}{c}\text { Path } \\
\text { planning } \\
\text { algorithm }\end{array}$ & $\begin{array}{l}\text { Path } \\
\text { length } \\
(\mathrm{cm})\end{array}$ & $\begin{array}{c}\text { The } \\
\text { number } \\
\text { of } \\
\text { passing } \\
\text { points }\end{array}$ & $\begin{array}{c}\text { time } \\
\text { consuming(S) }\end{array}$ & $\begin{array}{l}\text { Whether } \\
\text { the } \\
\text { results } \\
\text { are the } \\
\text { same }\end{array}$ & $\begin{array}{l}\text { Increas } \\
\text { e rate of } \\
\text { the path } \\
\text { length }\end{array}$ & $\begin{array}{l}\text { The reduction } \\
\text { rate of the } \\
\text { number of } \\
\text { passing points }\end{array}$ & $\begin{array}{l}\text { Comprehensiv } \\
\text { e results } \\
\text { (excellent or } \\
\text { bad) }\end{array}$ \\
\hline \multirow[b]{2}{*}{1} & \multirow{2}{*}{15} & $\mathrm{G}$ & 4156 & 80 & 0.0001 & yes & \multirow{2}{*}{$0 \%$} & \multirow{2}{*}{$0 \%$} & \multirow{2}{*}{ ACO } \\
\hline & & $\mathrm{ACO}$ & 4156 & 40 & 0.02 & no & & & \\
\hline \multirow{2}{*}{2} & \multirow{2}{*}{30} & $G$ & 2893 & 53 & 0.0001 & yes & \multirow{2}{*}{$1 \%$} & \multirow{2}{*}{$-1 \%$} & \multirow{2}{*}{ G } \\
\hline & & $\mathrm{ACO}$ & 2864 & 58 & 0.006 & no & & & \\
\hline \multirow{2}{*}{3} & \multirow{2}{*}{45} & $\mathrm{G}$ & 4578 & 89 & 0.0001 & yes & \multirow{2}{*}{$8.87 \%$} & \multirow{2}{*}{$6.74 \%$} & \multirow{2}{*}{ ACO } \\
\hline & & $\mathrm{ACO}$ & 4172 & 83 & 0.006 & no & & & \\
\hline \multirow{2}{*}{4} & \multirow{2}{*}{60} & $\mathrm{G}$ & 5077 & 108 & 0.0001 & yes & \multirow{2}{*}{$26.22 \%$} & \multirow{2}{*}{$31.50 \%$} & \multirow{2}{*}{$\mathrm{ACO}$} \\
\hline & & ACO & 3746 & 75 & 0.035 & no & & & \\
\hline \multirow{2}{*}{5} & \multirow{2}{*}{80} & $\mathrm{G}$ & 9262 & 243 & 0.0001 & yes & \multirow{2}{*}{$98 \%$} & \multirow{2}{*}{$0.4 \%$} & \multirow{2}{*}{ ACO } \\
\hline & & ACO & 8354 & 242 & 0.012 & no & & & \\
\hline \multirow{2}{*}{6} & & G & 10456 & 263 & 0.0001 & yes & & $281 \%$ & $A C O$ \\
\hline & 100 & $\mathrm{ACO}$ & 9420 & 256 & 0.04 & no & $9.91 \%$ & $2.81 \%$ & ACU \\
\hline & & $\mathrm{G}$ & 9962 & 253 & 0.001 & yes & & & \\
\hline 7 & 120 & ACO & 9081 & 242 & 0.01 & no & $8.85 \%$ & $4.23 \%$ & ACO \\
\hline & & $\mathrm{G}$ & 9540 & 260 & 0.0004 & yes & & $760 \%$ & $\mathrm{ACO}$ \\
\hline 8 & 150 & ACO & 7607 & 240 & 0.3 & no & $20.29 \%$ & $7.62 \%$ & $\mathrm{ACO}$ \\
\hline & & $\mathrm{G}$ & 10971 & 293 & 0.0001 & yes & & & \\
\hline 9 & 180 & ACO & 9991 & 286 & 0.025 & no & $8.93 \%$ & $2.49 \%$ & ACO \\
\hline & & $\mathrm{G}$ & 12476 & 333 & 0.0001 & yes & & & \\
\hline 10 & 200 & ACO & 10700 & 306 & 0.075 & no & $14.23 \%$ & $8.26 \%$ & ACO \\
\hline & & $\mathrm{G}$ & 12435 & 340 & 0.0001 & yes & & & \\
\hline 11 & 220 & ACO & 10845 & 315 & 0.075 & no & $12.79 \%$ & $7.24 \%$ & ACO \\
\hline & & G & 9396 & 254 & 0.0003 & yes & & & \\
\hline 12 & 230 & $\mathrm{ACO}$ & 8552 & 240 & 0.08 & no & $8.98 \%$ & $5.51 \%$ & ACO \\
\hline & & $\mathrm{G}$ & 11916 & 381 & 0.0008 & yes & & & \\
\hline 13 & 240 & $\mathrm{ACO}$ & 10637 & 363 & 0.25 & no & $10.73 \%$ & $4.72 \%$ & ACO \\
\hline & & $\mathrm{G}$ & 12183 & 396 & 0.0008 & yes & & & \\
\hline 14 & 260 & ACO & 10781 & 379 & 0.2 & no & $11.51 \%$ & $4.3 \%$ & ACO \\
\hline & & $\mathrm{G}$ & 12853 & 406 & 0.0007 & yes & & & \\
\hline 15 & 280 & $\mathrm{ACO}$ & 11153 & 381 & 0.6 & no & $13.23 \%$ & $6.16 \%$ & ACO \\
\hline & & $\mathrm{G}$ & 16445 & 456 & 0.0007 & yes & & & \\
\hline 16 & 308 & $\mathrm{ACO}$ & 13885 & 422 & 0.5 & no & $15.57 \%$ & $7.48 \%$ & ACO \\
\hline & & $G$ & 15648 & 438 & 0.0008 & yes & & & \\
\hline 17 & 320 & ACO & 13026 & 407 & 0.5 & no & $16.76 \%$ & $7.19 \%$ & ACO \\
\hline & & $\mathrm{G}$ & 15587 & 445 & 0.001 & yes & & & \\
\hline 18 & 350 & $\mathrm{ACO}$ & 12983 & 410 & 0.35 & no & $16.71 \%$ & $7.98 \%$ & ACO \\
\hline
\end{tabular}

From the four dimensions, this paper tested and compared them by the tasks of eighteen different groups, and the average result of 10 tests. It can be seen from Table 1 that in most cases the improved ant colony algorithm has the advantage over the Greedy algorithm from the aspects of path length and route points. Only when the number of task points is relatively small and the path of the task point is relatively simple, the path planning result of the improved ant colony algorithm is the same as the Greedy algorithm. In terms of the number of passing points, occasionally there will be more passing points for the improved ant colony algorithm. But the improved ant colony algorithm is always shorter than the Greedy algorithm in terms of path length. From the time consuming aspect, the improved ant colony algorithm is inferior to the Greedy algorithm, but the time efficiency is within one second. From the stability of path, Greedy algorithm is relatively more stable, and each time the results are the same. But the path planned by the improved ant colony algorithm is unstable, which is due to the randomness of the ant colony algorithm. Meantime the randomness is inevitable. They have their own advantages and disadvantages. Combined with the practical application of the laser inspection robot in the substation, the improved ant colony algorithm has the advantage over the Greedy algorithm from the comprehensive angle.

At the same time, this paper combines the grid method and the topology method to model environment and uses MATLAB dynamically simulate the algorithm proposed in this paper. The 
simulation results are shown in Figure 2. In figure 2, the black grid represents the obstacle, the white grid represents the accessible road, the red asterisk represents the undetected task point, the red dot represents the detected task point, and the black arrow represents the inspection path and the direction of the laser inspection robot. The laser inspection robot start to patrol from the starting point. When the robot encountered a random obstacle, it would return to the nearest detection point, and then re-planned inspection path and continued to inspect, and finally arrived the initial "starting point" and completed the inspection task. The dynamic simulation results show that this method can realize autonomous obstruction and re-plan inspection path. When the patrol robot encounter an obstacle, it can self-obstruction and self-detect whether task is completed. If the inspection task is not completed, it will re-plan the new inspection path for the remaining undetected task point, and so as to complete the inspection task.
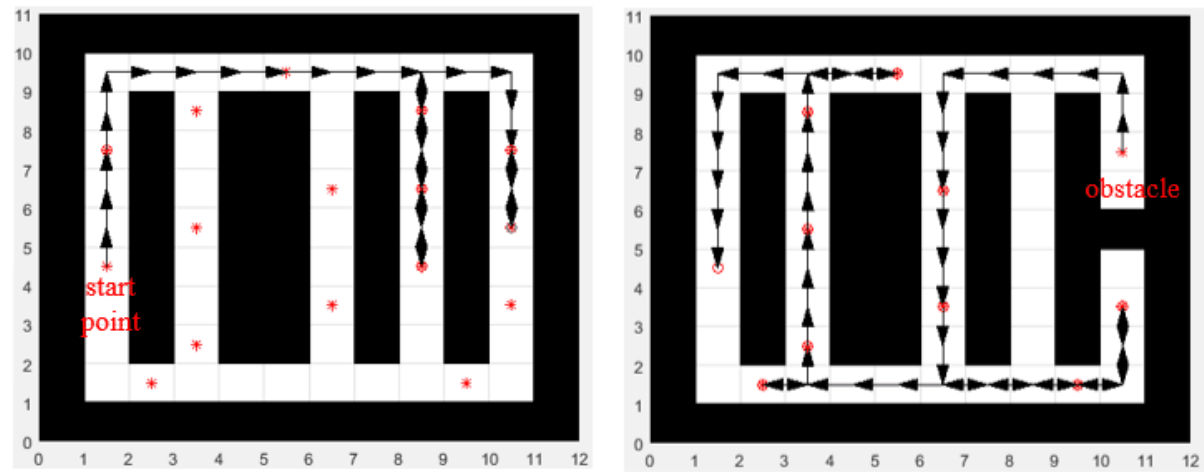

Figure 2. Dynamic Simulation Results of Global Path Planning Method with Independent Obstacle Avoidance

\section{Conclusion}

This paper presents a path planning algorithm that combined Dijkstra algorithm and improved ant colony algorithm. Firstly, use the topology method to model the environment based on the road information and the location information of the detection points in the substation. Secondly, the Dijkstra algorithm is used to calculate the shortest path between any two detection points, that is, local path planning, obtain the shortest distance matrix and nearest neighbor matrix. Thirdly, the improved ant colony algorithm is used to carry out the global path planning of the detection points and obtain the inspection sequence of the detection points. Finally, the nearest neighbor matrix is used to refine the inspection sequence. The algorithm can reset the inspection route according to the location of the obstacle after the obstruction, and ensure that all the inspection points can be tested and the route is optimal or sub-optimal. In this paper, the improved ant colony algorithm and Greedy algorithm are simulated by Visual Studio $\mathrm{C}++$. Contrast by test from four different angles, the improved ant colony algorithm is more advantageous than Greedy algorithm. Through the simulation test, the path plan algorithm of the paper overcomes the defects in other path planning algorithms, such as the longer path, more time-consuming, not suitable for large-scale TSP, easily fall into local optimal, can't re-plan mission path after dynamic real-time self-obstruction, and can't ensure the completion of inspection tasks. At the same time, the method of grid and topology method are used to simulate the environment and dynamic simulation by MATLAB. The algorithm has good applicability and robustness, and improves the intelligence of laser inspection robot.

\section{References}

[1]. Chen Yao, Chen A-lian, Li Xiang-dong, Chen Wei. (2015) Global path planning design of transformer substation intelligent inspection robot [J]. Shandong Science, 28(1):114-119.

[2]. Jang Yin-jie, Lv Xue-qin, Duan Li-wei. (2015) Grid genetic algorithm substation inspection robot path plan [J]. Science and Technology \& Innovation, (6):12-14. 
[3]. Shandong Electric Power Research Institute. (2003) Substation equipment inspection intelligent mobile robot [J]. Robot technique and application, (30):36-37.

[4]. Emilio Garcia-Fidalgo, Alberto Ortiz. (2015) Vision-based topological mapping and localization methods: A survey[J]. Robotics and Autonomous Systems. 64:1.20.

[5]. Chen Yao. (2015) Global path design and implementation for substation intelligent inspection robot [D]. Jinan: Shandong University, 5.24.

[6]. Deng Yan, Yang Guo-wei, Deng Cheng-jing, Qi Sui-ping. (2015) A Path Planning Algorithm and Application based on Improved Cellular Neural Network[C]. Information and Communication Technologies, 7.29:759-766.

[7]. Zhang Qian, Li Ming, Wang Xue-song. (2010) Global Path Planning Method of Mobile Robot in Uncertain Environment[C]. The 22nd China Control and Decision Conference. 1.1:4320-4324.

[8]. Wang Rui, Wang Jin-guo, Wang Na. (2015) Research on path planning of mobile robot based on improved artificial potential field[C]. Joint International Mechanical Electronic and Information Technology Conference (JIMET 2015), 1.1:1085-1088.

[9]. Feng Zhi-gang. (2008) Using Dijkstra method to carry out the path optimization of Logistics transportation system [D]. Guangzhou: Zhongshan University, 5.1.

[10]. Chen Wei, Jiang Yan. (2016) Improving ant colony algorithm and particle swarm to solve TSP problem [J]. Information technology, (5):162-165+170.

[11]. Zhu Xu, Han Zhi. A new hybrid algorithm for solving large scale TSP [J]. Chinese journal of engineering mathematics. 2007.24(5):923-926. 